\title{
Microstructure and magnetic properties of melt-spun Alnico-5 Alloys
}

\author{
Konrad Löwe $^{1 *}$, Michael Dürrschnabel ${ }^{2}$, Leopoldo Molina-Luna ${ }^{2}$, Rajasekhar \\ Madugundo $^{3}$, Bianca Frincu ${ }^{1}$, Hans-Joachim Kleebe ${ }^{2}$, Oliver Gutfleisch ${ }^{1}$ and George C. \\ Hadjipanayis $^{3}$ \\ ${ }^{1}$ TU Darmstadt, Department of Material- and Geosciences, Functional Materials,Alarich-Weiß-Str. 16, 64287 \\ Darmstadt, Germany \\ ${ }^{2}$ TU Darmstadt, Department of Material- and Geosciences,Geomaterial Science, Schnittspahnstraße 9, 64287 \\ Darmstadt, Germany \\ ${ }^{3}$ Department of Physics and Astronomy, University of Delaware, Newark, DE, USA
}

\begin{abstract}
The aim of this work is to investigate the effect of very fine grain sizes on the spinodal decomposition in the Alnico system. Commercial Alnico 5 was melted and melt-spun with varying copper wheel speeds, which led to a grain size of 1-2 $\mu \mathrm{m}$. This value was further reduced to sub-micrometer size by a small addition of Boron (1at.\%). The spinodal decomposition was induced through a two-step annealing treatment under magnetic field in the range of $600-900^{\circ} \mathrm{C}$. It was found that the size of the spinodal structures is not influenced much by increased wheel speeds but becomes smaller with the addition of Boron. However, the difference in coercivity between the samples with and without Boron is only 50 Oe $(4 \mathrm{kA} / \mathrm{m})$. To study the influence of the annealing treatment two sets of samples are compared, one with the highest coercivity(366 Oe / 29 $\mathrm{kA} / \mathrm{m}$ ) and the other one with lower coercivity (180 Oe / $14.5 \mathrm{kA} / \mathrm{m})$. We found with Scanning transmission electron microscopy Energy-dispersive X-ray spectroscopy (STEM EDX) a much sharper chemical interface between the $\alpha 1$ and $\alpha 2$ precipitates in the former sample, which we attribute to be the main reason for the higher coercivity.
\end{abstract}




\section{Introduction}

Permanent magnets are crucial components inmany commercial applications with the most important ones in motors and generators. Nowadays, the magnets with the highest energy content rely on rare earth elements, e.g. the Nd-Fe-B system. However, the recent rare-earth crisis calls for the development of rare-earth-lean/free permanent magnets $[1,2,3]$. A prominent example for non-rare earth magnets are the so-called Alnico magnets, which are the first modern permanent magnets discovered by Mishima [4]already in 1931. They do not contain rare-earths and have a relatively high energy product. The magnetic hardness originates from the shape anisotropy of FeCo-rich magnetic rods ( $\alpha_{1}$ phase) which are embedded in a non-magnetic NiAl-rich matrix ( $\alpha_{2}$ phase) [5]. This microstructure is obtained through spinodal decomposition after a special heat-treatment. However, the low coercivity $H_{\mathrm{c}}(<2 \mathrm{kOe})$ of Alnico magnets limits their use for high temperature applications. The theoretical value of $H_{\mathrm{c}}$ based on rod shape anisotropy is expected to be much higher ( $>7 \mathrm{kOe}$ ) [6]. Recent studies by Zhou et al.[7] revealed that the morphology of the rods is inhomogenous and the matrix is weakly magnetic, both of which can lead to the low coercivity. Sun et al. [8]reported the coercivity-spinodal structure relation in Alnico 5-7 and Alnico8 commercial magnets. A similar study was carried out by Xing et al. [9] in Alnico 9 type alloys. All of the above reports are on bulk commercial magnets.

It has been shown theoretically that there exists a critical wavelength $\lambda_{\mathrm{C}}$ (which corresponds to the grain size of the homogeneous initial alloy) below which the spinodal decomposition is suppressed[10].This value is approximately $100 \mathrm{~nm}$. Consequently, Akdogan et al.[11]could not induce spinodal decomposition in Alnico thin films with a grain size of $20 \mathrm{~nm}$. Above the critical wavelength the morphology of the spinodal features, and therefore the magnetic properties, are supposed to vary greatly with grain size. Regarding this issue, in the present work we varied the grain size in Alnico meltspun ribbons through varying copper wheel speeds and by a small addition of Boron (1 at.\%). We investigate the correlation between grain size, coercivity, and spinodal structures. 


\section{Experimental details}

A commercial Alnico5 magnet was used as sample material in this study. The magnet was melted and subsequently melt-spun. Samples with different grain sizes were prepared by varying the copper wheel speed of the melt-spinner from $5-60 \mathrm{~m} / \mathrm{s}$. To further reduce the grain size another set of samples was prepared with the addition of a small amount of B (1 at.\%), which was added prior to melting. Spinodal decomposition was induced by subjecting the as-spun samples to heat-treatment at temperatures in the range of $600-900{ }^{\circ} \mathrm{C}$. The ribbons were heat-treated under vacuum at temperatures 700 $900{ }^{\circ} \mathrm{C}$ (for 2-30 minutes) and then were cooled to $600{ }^{\circ} \mathrm{C}$ with a cooling rate $\sim 100$ $\mathrm{K} / \mathrm{min}$. The heat-treatment was continued at this temperature for 120 minutes. During the decomposition heat treatment the samples were under a magnetic field of $1 \mathrm{~T}$, applied along the length of the ribbons. The magnetic properties were measured with Quantum Design VersaLab vibrating sample magnetometer. Microstructural characterization of the ribbons was carried out by electron microscopy. A JEOL JSM 7600F scanning electron microscope operated at $15 \mathrm{keV}$ was used in electron backscattering (BSE) mode to image the surface microstructure. A $200 \mathrm{kV}$ Jeol JEM 2100F (scanning) transmission electron microscope (STEM)equipped with an Oxford X-max80 EDX detector was used to determine the microstructure on the nanometer scale. Forthe TEM studies the samples were demagnetized and mounted on $\mathrm{Cu}$ rings using $\mathrm{Ag}$-bonded epoxy. Ion thinning was done in a Gatan Dual Ion Mill Model 600using $\mathrm{Ar}^{+}$ions at $3.5 \mathrm{keV}$ and $0.5 \mathrm{keV}$ ion energies, respectively. Annular dark-field (ADF) scanning transmission electron microscope (STEM) images and energy-dispersive X-ray (EDX) spectra were obtained using a $0.7 \mathrm{~nm}$ spot size being a compromise between spatial resolution and EDX counts (detector dead time around 10\%). Quantification of EDX spectra was carried out using the Cliff-Lorimer k-factor method.

\section{Results and discussion}

Backscattered SEM images of as-spun ( 5 and $60 \mathrm{~m} / \mathrm{s}$ )and heat-treated samples at $900{ }^{\circ} \mathrm{C}$ for $30 \mathrm{~min}$ and aged at $600{ }^{\circ} \mathrm{C}$ for 2 hours are shown in figure 1 . By examining 
SEM images of several different regions of the ribbons a drastic reduction of the grain size is clearly visible, from $5-10 \mu \mathrm{m}$ to roughly $1 \mu \mathrm{m}$ and below in the samples produced at $5 \mathrm{~m} / \mathrm{s}$ and $60 \mathrm{~m} / \mathrm{s}$ copper wheel speed, respectively. After the heat treatment to induce the spinodal decomposition the grain size is almost doubled to around $10 \mu \mathrm{m}$ and $1-2 \mu \mathrm{m}$ in the ribbons produced at $5 \mathrm{~m} / \mathrm{s}$ and $60 \mathrm{~m} / \mathrm{s}$, respectively (not shown).

The developed spinodal structures within the grains are shown in the high resolution SEM images (figure 1, bottom).A typical interpenetrating block structure can be observed in our samples, which is in contrast tothe work by Akdogan et al. on Alnico thin films[11].As mentioned in the introduction, the grain sizein these filmswas only around $20 \mathrm{~nm}$ and a spinodal decompositioncould not be induced. The brighter block like shapes correspond toFeCo-rich $\alpha 1$ phase and the dark interspacing phase represents the AlNi-rich $\alpha 2$ phase. The spinodal structures have approximately the same size in both samples. It can be concluded that grain sizes in the range of several micrometers have only a weak influence on the morphology of the spinodal structures. The respective hysteresis loops of the optimally heat-treated ribbons show a $H_{\mathrm{c}}$ of 226 Oe for the $5 \mathrm{~m} / \mathrm{s}$ sample compared to $201 \mathrm{Oe}$ for the $60 \mathrm{~m} / \mathrm{s}$ ribbons (figure2). The reason for the small difference in $H_{\mathrm{c}}$ might be the slightly smaller spinodal features in the $5 \mathrm{~m} / \mathrm{s}$ sample and a changed aspect ratio of the FeCo needles.

Figure 3 shows the BSE SEM images of the cross-section of ribbons melt-spun at $60 \mathrm{~m} / \mathrm{s}$ without and with 1 at.\% B addition. The samples are in as-spun state (top) and after spinodal decomposition (bottom). The grain size decreased significantly withB addition to values below $1 \mu \mathrm{m}$. Also, from the high resolution SEM images it can be seen that the spinodal morphology in the ribbons with B is significantly finer compared to that of the ribbons without B. Also, with B a minor secondary phase is formedwith a size about $50 \mathrm{~nm}$ (black dots seen in the figure), which we found to be Ti-rich with STEM EDX. Hysteresis loops of the heat-treated ribbons with and without $\mathrm{B}$ are shown figure 4.The $H_{\mathrm{c}}$ values of the samples with and without B are 255and $201 \mathrm{Oe}$, respectively. The reasons for the difference in coercivity might be the finer spinodal morphology in the B-containing sample or the occurrence of more secondary phases.

Summarizing theresults of the above two experiments:The grain size of Alnico ribbons was decreased by an order of magnitude by increasing the copper wheel speed from $5 \mathrm{~m} / \mathrm{s}$ to $60 \mathrm{~m} / \mathrm{s}$ to values, from $5-10 \mu \mathrm{m}$ to about $1 \mu \mathrm{m}$. The ribbons with bigger 
grains have a slightly finer spinodal morphology than the smaller grained ribbons, although the difference is not pronounced. It seems that in the range of $\mu \mathrm{m}$ the grain size does not strongly influence the size of the spinodal structures. When the grain size was decreased by B addition, a significantly finer spinodal structure developed compared to samples without B. Consequently, the coercivity of the B containing samples is higher compared to the ribbons without B. However, this difference could also be due to the difference in chemical composition and amount of secondary phases than with grain size.

Details of the spinodal structure arefurther investigated by High angle annular dark field (HAADF)-STEM and STEM-EDX studies. Two samplesof Alnico5 melt-spun at the same wheel speed of $26 \mathrm{~m} / \mathrm{s}$, but heat-treated at different conditions, having best (Alnico+)and worst (Alnico-) $H_{\mathrm{c}}$ values are selected for this study. The hysteresis loops of the ribbon samples heat-treated at $800{ }^{\circ} \mathrm{C}$ for $4 \mathrm{~min}\left(\right.$ Alnico + ) and at $800{ }^{\circ} \mathrm{C}$ for $30 \mathrm{~min}$ (Alnico-) and then aged at $600{ }^{\circ} \mathrm{C}$ for $120 \mathrm{~min}$ are shown in figure 5. The coercivities differ greatly between 366 and 180 Oe for Alnico+ and Alnico-, respectively. HAADFSTEM images ofboth ribbons are shown in figure 6. The images show spinodal structures precipitating along the magnetic field direction. The dark spots seen are found to be Tirich precipitates. It was mentioned before that in the samples with B addition more of these precipitates can be found.The morphology of the spinodal structures shows distinct differences between the two samples. While the spacing of the lamellae ( $\alpha 1$ phase) is roughly the same there is more matrix phase ( $\alpha 2$ phase) in Alnico-, i.e. the $\alpha 1$-lamellae are thinner. Compositional profiles across the phases have been recorded withEDX linescans perpendicular to the longitudinal direction of the lamellae (figure 6, bottom). The FeCo-rich lamellae and the NiAl-rich matrix phase can be clearly distinguished for both the samples. The difference in the concentrationsacross the phases,i.e. the ratio of peak and valley is more pronouncedin Alnico+ sample than in Alnicosample.Compositional profiles in Alnico+ also depict plateau like regions for $\mathrm{Fe}$ and $\mathrm{Co}$ unlike the sharp peaks and valleys in Alnico-. In other words, the chemical interface between both phases is sharp in Alnico+ and less defined in Alnico-. The quantitative composition of the two phases was measured with EDX point scans and is shown in Table 1.

To get a first estimate for the coercivity of Alnico a well-established relation for the coercivity of a fully aligned array of single domain particles is commonly used [5]: 


$$
H_{C}=(1-p)\left(N_{z}-N_{x}\right) M_{S}
$$

Here $\mathrm{p}$ is the volume fraction of the FeCo-rich al-lamellae, $\left(N_{z}-N_{x}\right)$ denotes the difference in demagnetization factors between the principal directions of the lamella (i.e. the shape anisotropy contribution) and $M_{S}$ is the saturation magnetization of the $\alpha 1$ lamellae. $M_{S}$ will increase with increasing content of $\mathrm{Fe}$ and $\mathrm{Co}$, consequently the coercivity should be proportional to the Fe and Co concentration in the $\alpha 1$-phase. Since they are, within the error limits, almost the same for both samples we doubt that they are responsible for the relatively big difference in coercivity. However, the formula was derived for an ideal separation of the magnetic phases. This separation is regarded as a necessity for a high coercivity and therefore it is likely that the main reason for the decreased coercivity is the less defined chemical interface rather than the absolute change in composition.Similar conclusions were drawn by Xing et al. [9] in commercial Alnico 5-8 magnets and by Sun et al. [12] in commercial Alnico 9 magnets. The other influencing factors, the volume fraction $p$ and the shape anisotropy $\left(N_{z}-N_{x}\right)$ are hard to precisely determine from our STEM images and EDX line scans but seem to be rather equal.

\section{Conclusions}

The influence of the microstructure on spinodal structures and the magnetic properties of melt-spun Alnico ribbons have been studied.The grain size of the ribbons was decreased from $5-10 \mu \mathrm{m}$ to about $1 \mu \mathrm{m}$ by increasing the copper wheel speed from 5 $\mathrm{m} / \mathrm{s}$ to $60 \mathrm{~m} / \mathrm{s}$, and further decreased by addition of 1 at.\%B to less than $1 \mu \mathrm{m}$. The size of the spinodal features was about the same in the ribbons without $\mathrm{B}$, which means that grain size above a certain threshold (which was theoretically shown to be $>100 \mathrm{~nm}$ in another work [10]) does not influence the spinodal decomposition anymore. Through addition of B the spinodal features became significantly smaller. This, however, resulted only in a small increase in coercivity of about 50 Oe.The coercivities obtained in all samples are about half of those in anisotropic bulk samples, eventhough the aspect ratio of spinodals are the same as in bulk [8,9]. In a second part we set out to compare an optimally heat treated sample with high coercivity with one with low coercivity. With 
STEM EDX linescans a much sharper compositional contrast between the $\alpha 1$ lamellae and the $\alpha 2$ matrix phase was found in the former sample, which we attribute to be the main reason for the higher coercivity.

\section{Acknowledgments}

Part of the work was supported by DOE BES DE-FG02-04ER4612.The authors also acknowledge financial support from the Hessen State Ministry of Higher Education, Research and the Arts via LOEWE RESPONSE.The Jeol JEM 2100F transmission electron microscope used in this work was partially funded be the German Research Fundation (DFG/INST163/2951). 


\section{References}

[1] M J Kramer, R W McCallum, I A Anderson, and S Constantinides, JOM, no. 64, p. $752,2012$.

[2] O Gutfleisch et al., Adv. Mater., no. 23, p. 821, 2011.

[3] M D Kuz'min, K P Skokov, H Jian, I Radulov, and O Gutfleisch, J Phys: Cond Mat, no. 26, p. 064205.

[4] T Mishima, Stahl U. Eisen, no. 53, p. 79, 1931.

[5] R A McCurrie, Structure and Properties of Alnico Permanent Magnet Alloys. In E.P Wolfarth (Ed.) Ferromagnetic Materials 3. UK: North-Holland Publishing Company, 1982.

[6] L Zhou et al., Metall. Mater. Trans., no. E1, p. 27, 2014.

[7] L Zhou et al., Acta Mater, no. 74, 2014.

[8] Y L Sun et al., J. Magn. Magn. Mater., no. 379, p. 58, 2014.

[9] Q Xing et al., IEEE Trans. Magn., no. 49, p. 3314, 2013.

[10] T A Abinandanan and H Ramanarayan, Bull. Mater. Sci., no. 26, p. 189, 2003.

[11] O Akdogan, L Wanfeng, and G Hadjipanayis, J Nanopart Res, no. 14, p. 891, 2012.

[12] X Y Sun et al., J. Magn. Magn. Mater., no. 348, p. 27, 2013. 


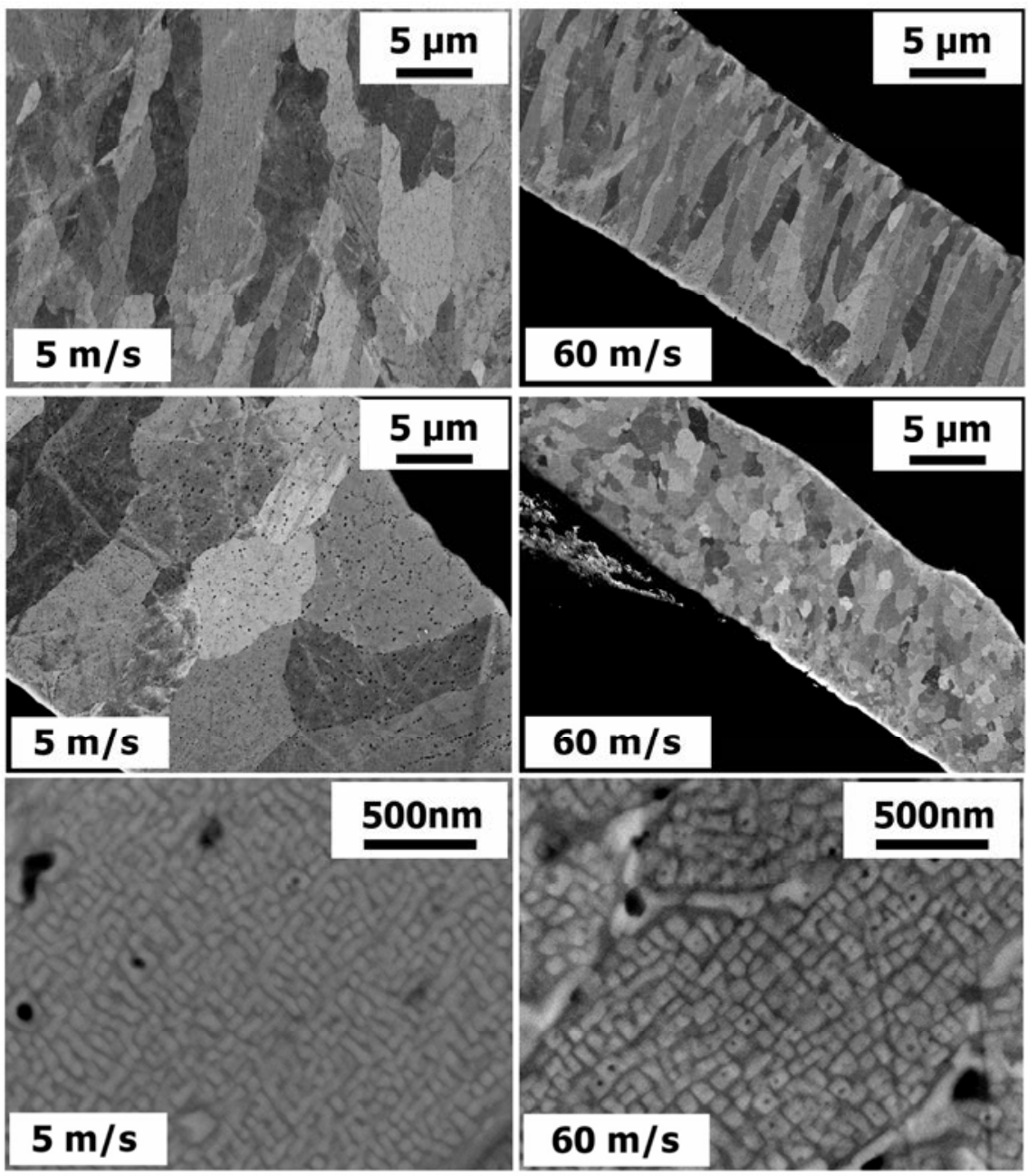

Figure 1: BSE SEM images of microstructures observed from the side of the ribbons(top) and from the cross-section (middle) of as-spun samples prepared at $5 \mathrm{~m} / \mathrm{s}$ and $60 \mathrm{~m} / \mathrm{s}$ wheel speed. Spinodal structures in the same ribbons after subsequent heat-treatment (bottom, seen from cross-section). 


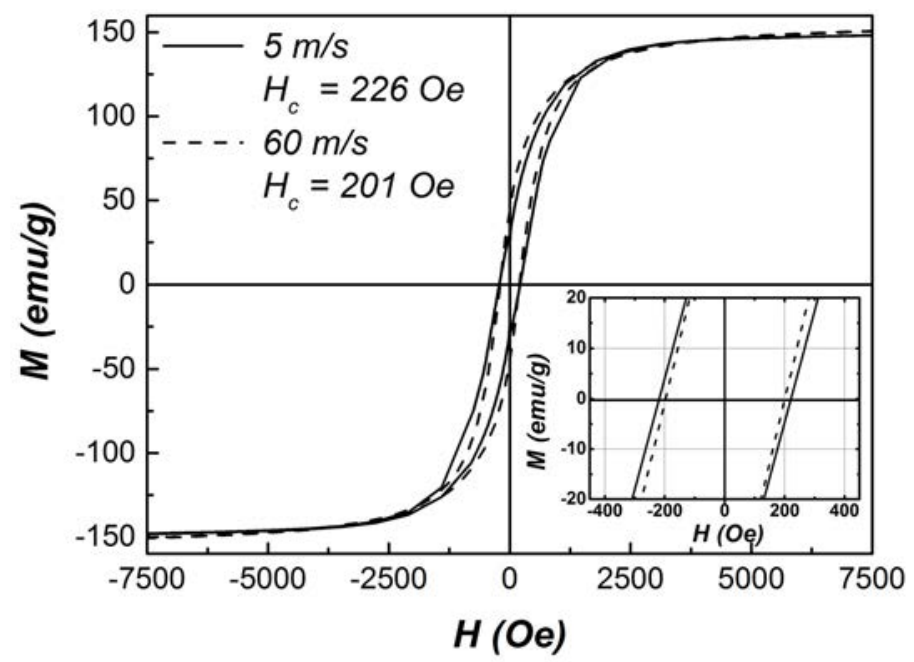

Figure 2: Hysteresis loops of the samples melt-spun at 5 and $60 \mathrm{~m} / \mathrm{s}$ wheel speeds after spinodal decomposition. 


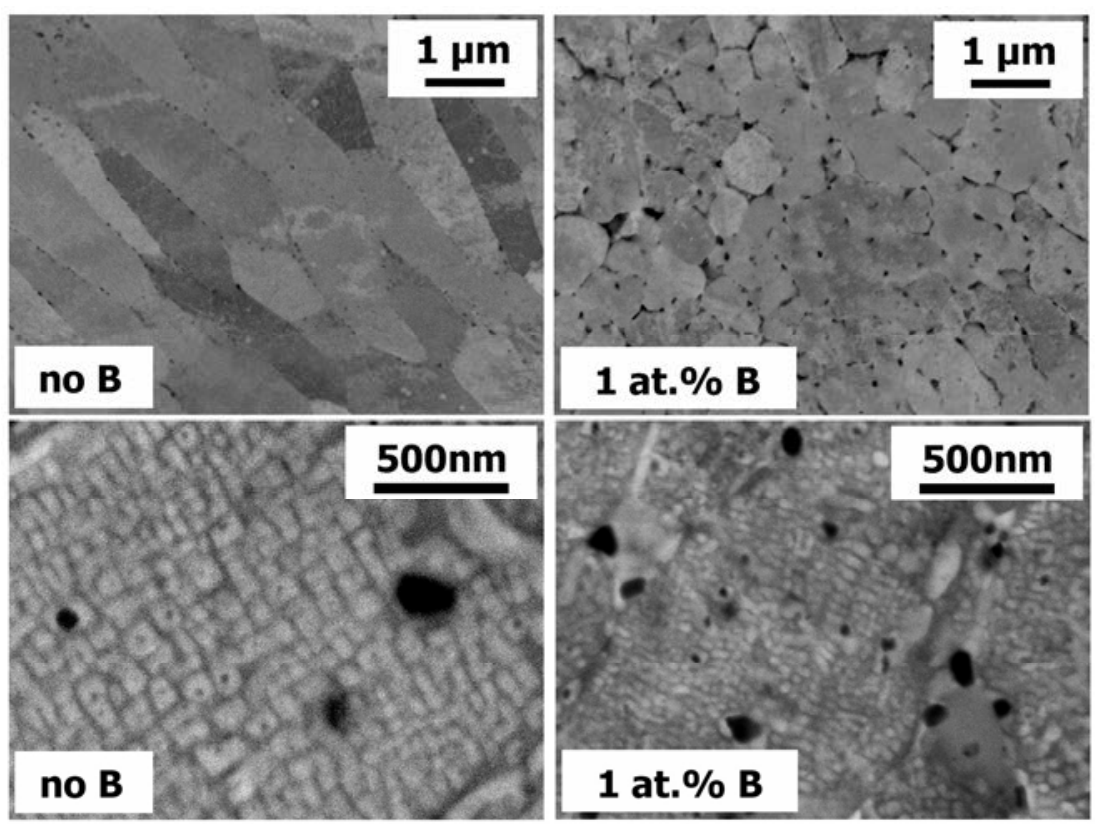

Figure 3: (Top) BSE SEMimages taken from the cross section of the as-spun samples without B and with 1 at.\% B addition. (Bottom) Spinodal structures in the same ribbons after heat-treatment. 


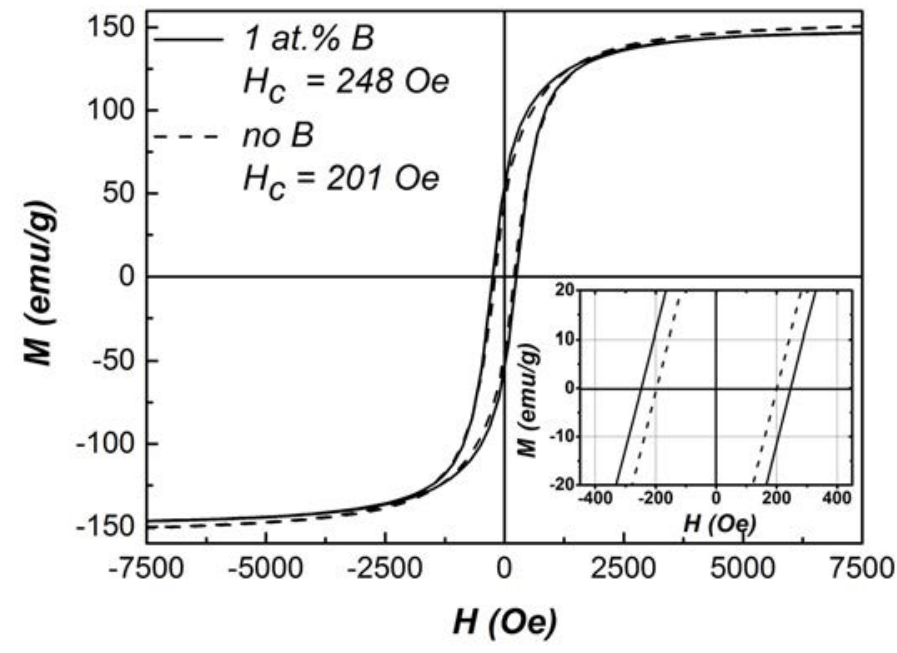

Figure 4: Comparisons of hysteresis loops for samples with and without the addition of 1 at. $\%$ B produced at $60 \mathrm{~m} / \mathrm{s}$ after spinodal decomposition. 


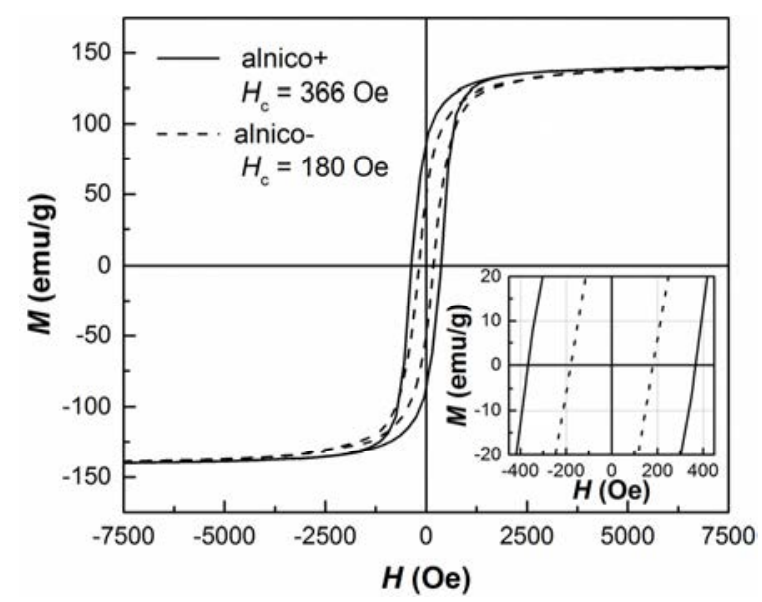

Figure 5: Hysteresis loops of Alnico $+\left(800{ }^{\circ} \mathrm{C}\right.$ for 4 minutes then $600{ }^{\circ} \mathrm{C} 120$ minutes; $H_{\mathrm{c}}$ $=366 \mathrm{Oe})$ and Alnico- $\left(800{ }^{\circ} \mathrm{C}\right.$ for 30 minutes then $600{ }^{\circ} \mathrm{C} 120$ minutes; $\left.H_{\mathrm{c}}=180 \mathrm{Oe}\right)$ ribbons. 

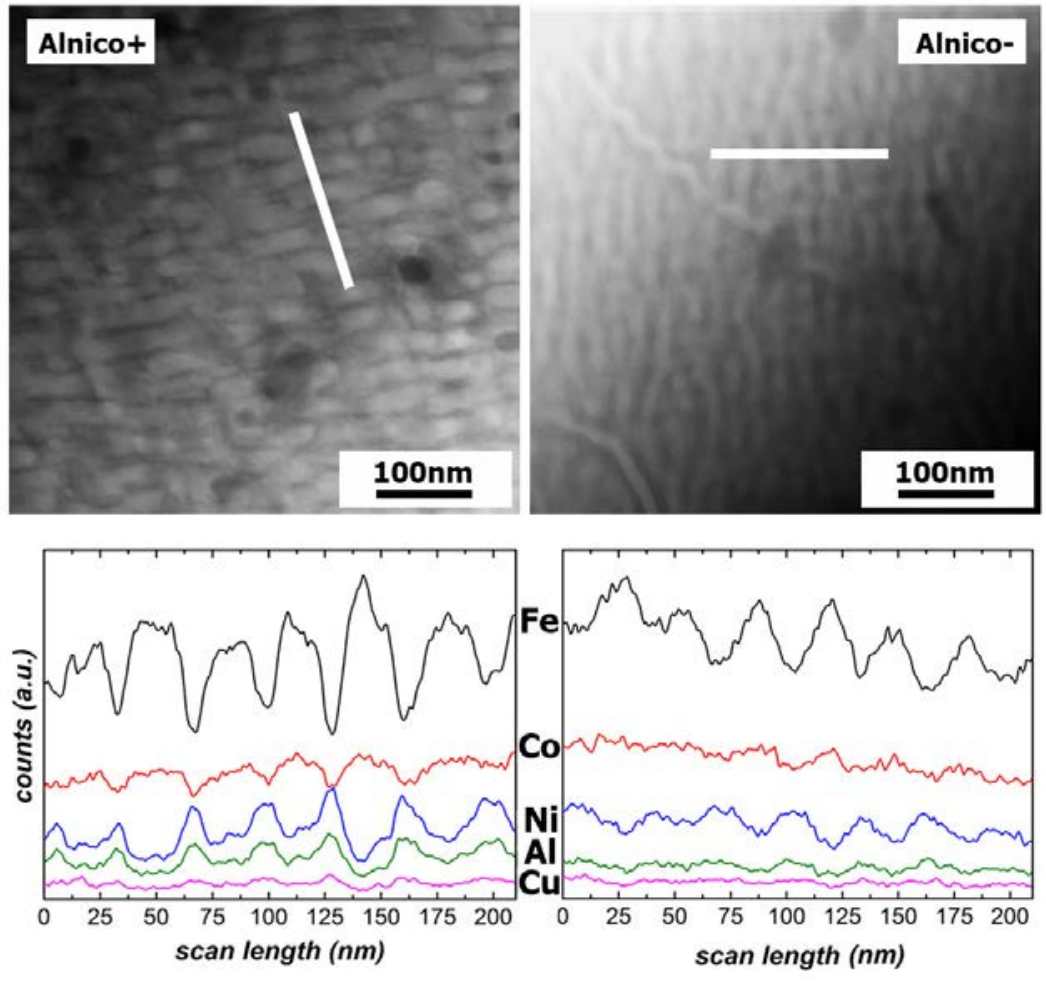

Figure 6: Differences in microstructure and chemical composition in high coercivity Alnico+ and low coercivity Alnico- (cp. figure 5). 
Table 3: Composition of lamellae and matrix phase in Alnico+ and Alnico- measured by EDX (in at.\%). Note that since a $\mathrm{Cu}$ ring was used for mounting the sample the measured $\mathrm{Cu}$ concentration might be slightly too high due to stray radiation.

\begin{tabular}{|c|c|c|c|c|c|c|}
\hline \multirow{2}{*}{ Sample } & \multicolumn{2}{|c|}{ Lamella } & \multicolumn{2}{c|}{ Matrix } & \multicolumn{2}{c|}{ Difference Lamella - Matrix } \\
\cline { 2 - 7 } & Alnico+ & Alnico- & Alnico+ & Alnico- & Alnico+ & Alnico- \\
\hline $\mathrm{Al}$ & $14.5 \pm 2.5$ & $10.9 \pm 2.2$ & $23.9 \pm 3.0$ & $17.1 \pm 1.0$ & $9.4 \pm 3.9$ & $6.2 \pm 2.4$ \\
\hline $\mathrm{Fe}$ & $50.3 \pm 4.4$ & $53.2 \pm 3.9$ & $34.5 \pm 5.3$ & $38.4 \pm 2.4$ & $15.8 \pm 6.9$ & $14.8 \pm 4.6$ \\
\hline $\mathrm{Co}$ & $21.6 \pm 0.7$ & $22.8 \pm 0.6$ & $19.1 \pm 0.8$ & $21.6 \pm 0.3$ & $2.5 \pm 1.1$ & $1.2 \pm 0.7$ \\
\hline $\mathrm{Ni}$ & $10.0 \pm 2.1$ & $10.0 \pm 1.9$ & $17.1 \pm 2.4$ & $17.8 \pm 1.4$ & $7.1 \pm 3.2$ & $7.8 \pm 2.4$ \\
\hline $\mathrm{Cu}$ & $2.3 \pm 0.4$ & $2.1 \pm 0.5$ & $4.2 \pm 1.4$ & $3.7 \pm 0.6$ & $1.9 \pm 1.5$ & $1.6 \pm 0.8$ \\
\hline
\end{tabular}

